\title{
Age-Related Alterations in Gait Function in Freely Moving Male C57BL/6 Mice:Translational Relevance of Decreased Cadence and Increased Gait Variability
}

\author{
Stefano Tarantini, PhD, ${ }^{1,2}$ Andriy Yabluchanskiy, MD, PhD, ${ }^{1,2}$ Gábor A. Fülöp, MD, ${ }^{1-3}$ \\ Tamas Kiss, MD, ${ }^{1}$ Aleksandra Perz, MS, ${ }^{1}$ Daniel O'Connor, ${ }^{1}$ Emily Johnson, ${ }^{1}$ Farzaneh Sorond, \\ MD, PhD, ${ }^{4}$ Zoltan I. Ungvari, MD, PhD, ${ }^{1,2,5}$ and Anna Csiszar, MD, PhD 1,2,5,*
}

${ }^{1}$ Vascular Cognitive Impairment and Neurodegeneration Program, Center for Geroscience and ${ }^{2}$ Translational Geroscience Laboratory, Reynolds Oklahoma Center on Aging, Department of Geriatric Medicine, University of Oklahoma Health Sciences Center. ${ }^{3}$ Division of Clinical Physiology, Faculty of Medicine, University of Debrecen, Hungary. ${ }^{4}$ Department of Neurology, Northwestern University, Chicago, Illinois. ${ }^{5}$ Department of Medical Physics and Informatics, Faculty of Medicine and Faculty of Science and Informatics, University of Szeged, Hungary.

*Address correspondence to: Anna Csiszar, MD, PhD, Reynolds Oklahoma Center on Aging, Department of Geriatric Medicine, University of Oklahoma Health Sciences Center, 975 NE 10th Street, BRC 1303, Oklahoma City, OK 73104. E-mail: anna-csiszar@ouhsc.edu

Received: July 5, 2018; Editorial Decision Date: October 11, 2018

Decision Editor: Rozalyn Anderson, PhD

\begin{abstract}
Age-related gait dysfunction and balance disorders are a major cause of falls and injury in the elderly population. Epidemiological studies have shown that disturbances in gait coordination which manifest with age are associated with increased morbidity and mortality, impaired cognitive capacity, as well as reduced level of function and loss of independence. In geroscience, mice are the most frequently used model system to test efficiency of antiaging interventions. Despite the clinical importance of age-related gait abnormalities in older adults, the impact of aging on mouse gait coordination is not well documented in the literature. To characterize the effect of aging on mouse gait, we assessed gait function in young (3-monthold) and aged (24-month-old) freely moving C57BL/6 mice using the semiautomated, highly sensitive CatWalk XT system for quantitative assessment of footfall and motor performance. We found that aged mice exhibited significantly decreased cadence and increased stride time variability. Aging also tended to alter footfall patterns. In aged mice, speed, swing speed, stride length, duty cycle, base of support, terminal dual stance, the regularity index, and the gait symmetry index were unaltered. Thus, aging is associated with characteristic alterations in gait function in C57BL/6 mice, which could potentially be assessed as clinically relevant endpoints in geroscience studies testing the effects of antiaging interventions.
\end{abstract}

Keywords: Gait, Mobility, Cognitive decline, Brain aging

A hallmark of human aging is the decline of gait function (1-3). Decline in gait performance is highly prevalent in the aging population; in fact, over one in every three individuals aged 70 years or older exhibits some form of gait abnormality, which is a major cause of functional impairment. Importantly, age-related decline in gait function increases susceptibility of the elderly population to fall-related injuries (2). More than $30 \%$ of people over 65 years of age fall each year and in half of the cases falls are recurrent (4). Falls often result in serious injuries such as hip fracture, subdural hematoma, traumatic brain injury, and premature nursing home admissions (5). Further, in humans, measures of gait, balance, and coordination are commonly used to predict future age-related morbidity and mortality (6). Importantly, gait abnormalities can predict cognitive impairment in the elderly population and in patients with neurodegenerative disease (7). In the field of gerontology, the emerging concept of frailty is described as the decrease in physiological functional reserves which associates with the increased risks of disability, institutionalization, unplanned hospitalization, falls with or 
without trauma, and early death (8). Recent studies have demonstrated that the intimate association between the age-related changes in gait coordination and the increase in frailty can be exploited to develop clinically relevant screening tests to guide the implementation of a comprehensive geriatric assessment in the elderly population (9).

Over the past decade a wide range of genetic, pharmacological, and nutritional interventions were identified, which increase the life span of laboratory mice. However, it became apparent that to evaluate the translational potential of any proposed antiaging intervention, information on how that particular intervention alters the health span of mice is desperately needed (10). Preliminary evidence suggests that mouse gait is altered in pathophysiological conditions associated with accelerated brain aging (11). Despite these advances and the emerging clinical importance of the relationship between human gait and aging, the effects of aging on mouse gait are not well characterized.

This study was designed to provide a comprehensive analysis of age-related changes in gait characteristics in C57BL/6 mice (the most widely used murine aging model), to identify translationally relevant preclinical measurements of physiological dysfunction. To achieve that goal, we compared gait function in freely moving 3- and 24-month-old mice using a highly sensitive computer-aided system for quantitative assessment of footfall and motor performance. We analyzed gait parameters that have direct translational relevance (eg, gait speed, swing speed, cadence, stride length, stride time, and base of support) and adopted and developed methods to analyze stride time and stride length variability, which are considered sensitive indices of age-related human gait abnormalities with predictive potential.

\section{Methods}

The detailed Methods section for this study is available as Supplementary Material.

\section{Analysis of Gait Function}

To determine the impact of aging on gait coordination, we tested young (3-month-old) and aged (24-month-old) male C57BL/6J mice ( $n=20$ in each group) using a highly sensitive, automated computerassisted method (CatWalk; Noldus Information Technology Inc) as described (11). All procedures were approved by the Institutional Animal Care and Use Committee (IACUC) of the University of Oklahoma Health Sciences Center.

Mean gait characteristics calculated included speed, swing speed, cadence, stride length, stride time, duty cycle, base of support, terminal dual stance, and the regularity index, which is a fractional measure of inter-paw coordination (11).

Investigating gait variability, the stride-to-stride fluctuations in gait parameters, offers a sensitive, novel method of quantifying subtle changes in locomotion in mice. Step time and step length variability were analyzed by computing the median absolute deviation (MAD) for data sets that contained more than 200 steps for each animal obtained in consecutive runs at similar speeds where each step value is $X\left(X_{1}, X_{2}, \ldots, X_{200}\right)$. MAD is a robust measure of statistical dispersion, which is more resilient to outliers in a data set than the standard deviation:

$$
\operatorname{MAD}=\operatorname{median}\left(\left|X_{i}-\operatorname{median}(X)\right|\right)
$$

To assess gait symmetry on the basis of spatial-temporal gait characteristics, the symmetry index (SI) was used:

$$
\mathrm{SI}=\frac{\left|X_{\text {Left }}-X_{\text {Right }}\right|}{0.5 \times\left(X_{\text {Left }}+X_{\text {Right }}\right)} \times 100 \%
$$

\section{Statistical Analysis}

Statistical analysis was carried out by unpaired $t$ test using Prism 5.0. A $p$ value less than .05 was considered statistically significant. Data are expressed as mean \pm SEM.

\section{Results}

\section{Effects of Aging on Gait Coordination in Mice}

In this study, there was no statistically significant difference in walking speed, stride time, and swing speed between freely moving young and aged mice. We also did not observe differences between young and aged mice in stride length, duty cycle, base of support, and terminal dual stance (Supplementary Figure 1AG). Aging significantly decreased mouse cadence (Figure 1B) paralleling the human findings that stepping rate is decreased with the progression of age (12).

The regularity index, a comprehensive measure of inter-paw coordination, was unaffected by aging (Figure 1A). The study of gait variability, the stride-to-stride fluctuations in walking, offers a sensitive method of quantifying and characterizing locomotion. Previous studies in older adults suggest that measures of gait variability are more closely related to cognitive decline or falls than other measures based on the mean values of other gait characteristics (1). In this study in aged mice, a trend for increased stride length variability was discernible (Figure 1C). We found a statistically significant increase in stride time variability with advanced aging (Figure 1D).

Gait involves a cyclical and laterally alternating stride progression to maintain balance. Comparing left versus right stride lengths and stride time asymmetries may reflect potential functional deficits that may otherwise go unnoticed. The left-right stride characteristics were graphically compared (Figure $1 \mathrm{E}$ and $\mathrm{F}$ ) and a lateralization index was calculated (see Supplementary Methods). We found that aged mice have a significantly more asymmetric stride pattern during gait compared to young (Figure 1G) and a tendency to exhibit increased asymmetry in stride time (Figure $1 \mathrm{H}$ ). Another sensitive measure of gait symmetry is the SI (13); we found that stride length and stride time SIs tended to increase with age but do not reach statistical significance (Figure $1 \mathrm{I}$ and J). The regularity index was similar in young and aged animals (Figure 1A), suggesting that both groups used predominantly the four natural gait patterns (see Supplementary Methods). Yet, we found age-related differences in the gait-pattern frequency distribution (Figure 2). Aged mice exhibited a $50 \%$ increase in frequency of the radial pattern AA, known as the "giraffe walk." Alternating contralateral footfall patterns $(\mathrm{CA} / \mathrm{CB})$ were used with lesser frequency in aged mice compared to young controls. Thus, the age-related adjustments in gait mechanics, observed via gait-pattern frequency distribution, revealed that aging was accompanied by a preferential reliance on the AA radial pattern over the alternating $\mathrm{AB}$ and $\mathrm{CB}$ gait patterns that instead associated with young age in mice.

\section{Discussion}

This study provides evidence that aging per se induces alterations in gait function in a widely used and well-characterized mouse model of aging. Changes in gait characteristics were studied in 24-monthold mice, whose age is biologically equivalent of an approximately 70-year-old human. At this biological age, cognitive dysfunction is evident in mice (14), similar to humans, whereas the age-related, 

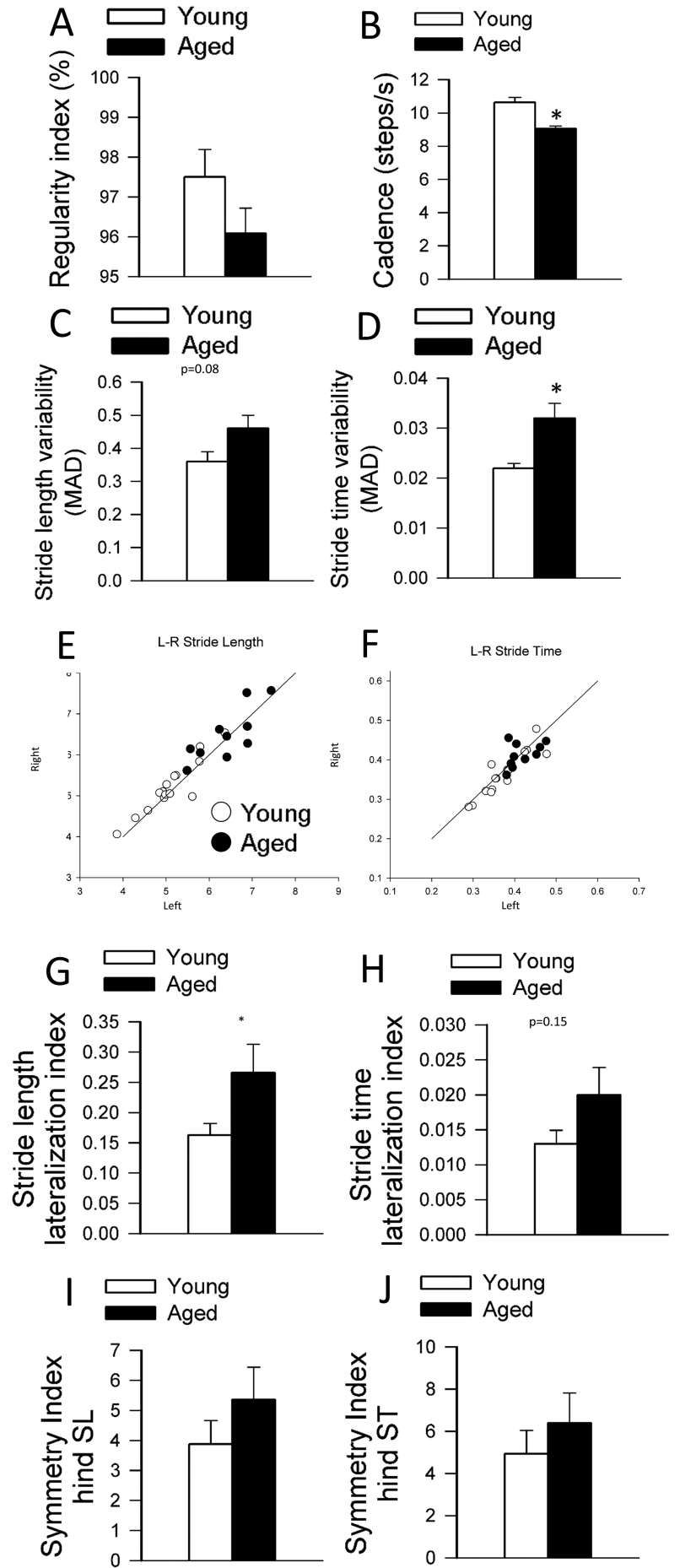

Figure 1. Effects of aging on gait regularity, cadence, gait variability, and gait symmetry in C57BL/6 mice. (A) Regularity index remains unchanged in aged mice as compared to spontaneously walking control C57BL/6 mice. (B) Cadence. (C) Stride length variability. (D) Stride time variability significantly increases in aged mice compared to young control. MAD: median absolute deviation. (E) Relationship between left and right stride lengths and (F) stride times in young and aged mice. (G) Stride length lateralization index. Aged animals show significantly weaker correlation in the sidedness between their stride lengths. $(\mathrm{H})$ Stride time lateralization index. (I) Gait symmetry indices were calculated for hind paws stride length (SL) and (J) stride times (ST). Data are mean \pm SEM. ${ }^{*} p<.05$ using student's $t$-test.

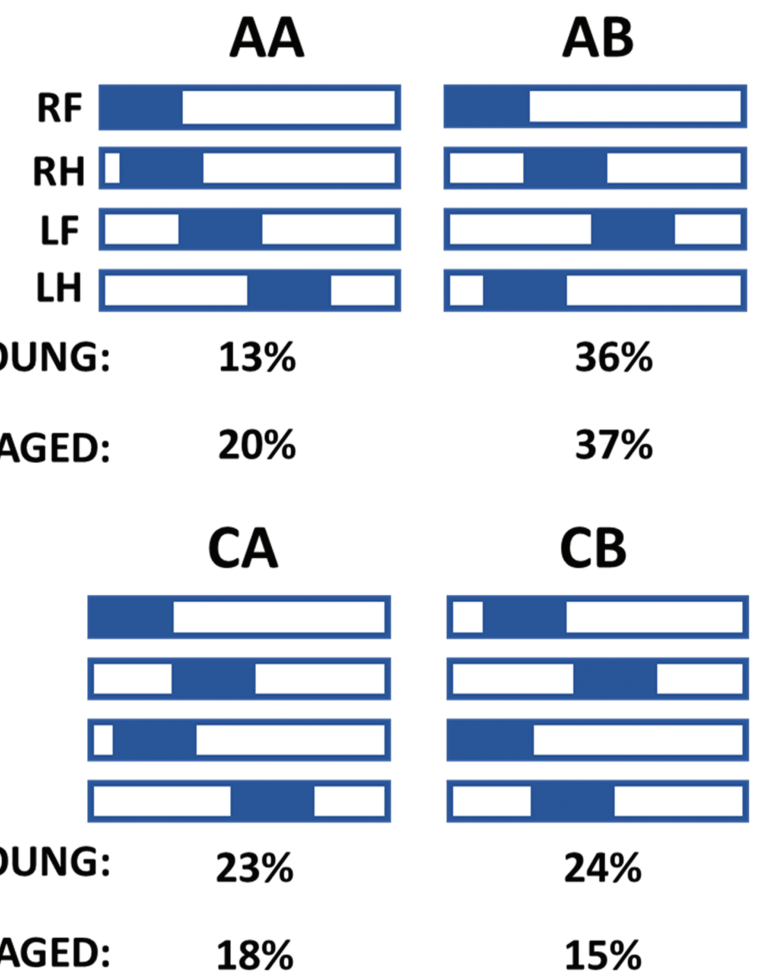

Figure 2. Age-related impairment of gait coordination results in altered gaitpattern distribution in C57BL/6 mice. Hildebrand plot of the common gait patterns: AA (RF-RH-LF-LH), AB (LF-RH-RF-LH), CA (RF-LF-RH-LH), and CB (LF-RF-LH-RH). Percentages indicate relative use of each step pattern in each mouse age group. The most common step pattern in both was the AB pattern. Aged mice used more frequently the radial $A A$ pattern than young control mice, and compensated with a decreased use of the alternating $A B$ pattern and $\mathrm{CB}$ pattern.

species-specific comorbidities that may affect mouse gait (ie, tumor susceptibility and cancer-related muscle wasting and frailty) are not yet prominent. Using automated, computer-aided gait analysis, we demonstrated that 24-month-old freely moving C57BL/6 mice exhibited significantly altered gait signatures compared to 3-month-old controls, including changes in cadence, gait variability, and footfall pattern distribution. These findings reflecting mild alterations in gait function at this age provide important reference values for the design of future studies attempting to characterize the effects of dietary, pharmacological, or genetic interventions on mouse health span in geroscience research. Future studies are warranted to assess changes in gait parameters in later stages of life span and to determine sex-specific effects of aging on gait function in mice. Recently, a number of new methodologies have been developed to assess frailty as a measure of health span in aging mice with both a frailty index approach (15) and a frailty phenotype approach (16). Incorporation of gait variability measures in frailty assessment may be considered in future studies.

Our findings also have important translational relevance. Gait dysfunction and balance disorders are a pervasive phenomenon of human aging. Clinical studies demonstrate persistent reductions in gait speed and stride length (1-3) and increases in gait variability (17) in older individuals when compared to younger adults. Agerelated changes in gait coordination can lead to falls and associate with increased morbidity and mortality, as well as reduced level of independence in the aging population. The mechanisms underlying age-related changes in gait function in older adults and aged mice 
are likely multifaceted (18). Much is known about the contributions to age-related mobility problems due to cardiopulmonary, musculoskeletal, and overt neurological conditions (eg, stroke and Parkinson's disease). In contrast, much less is known about the age-related mechanisms affecting central neural control of mobility in older adults without overt neurological diseases, which promote subclinical gait abnormalities. Increasing evidence supports the hypothesis that many senile gait abnormalities represent an early manifestation of subclinical brain pathologies because their occurrence has been shown to associate with increased risk of cognitive decline and/or dementia, including Alzheimer's disease, and death (1). Thus, there is an urgent need for preclinical studies that differentiate aging from disease-related processes, focusing on central nervous system alterations, which may be amenable to intervention. Mouse models of aging are expected to be used extensively to understand the relationship among neuroinflammation and other pathological processes, altered brain networks, and compromised gait function. Preclinical gait research on mouse models of aging will likely promote the development of effective interventions for mobility limitations and the subsequent reduction of a major functional disability for older adults.

There is emerging evidence that in aging there is an accumulation of nonspecific microvascular abnormalities that are distributed across the brain, including blood-brain barrier disruption and consequential neuroinflammation, impaired neurovascular coupling, impaired autoregulation, and microvascular injury, all of which have been linked to impaired higher brain function including cognitive decline (19). A particularly exciting field of research focuses on understanding the sequence of events and causal pathways that link these early cerebromicrovascular alterations to gait abnormalities. Data from the MOBILIZE Boston study (3) establish a causal relationship between age-related decrease in functional hyperemia and altered gait function in humans. Recent mechanistic preclinical studies in mice provide proof of concept that impaired neurovascular coupling responses are causally linked to gait abnormalities, including an increased gait variability $(11,20)$. Preclinical data obtained in aged mice also provided recently the first evidence that development of cerebral microhemorrhages can also cause subclinical gait abnormalities (21). Recently, hypotheses were put forward that interventions that prevent and/or reverse age-related microvascular alterations may improve gait function. Recent findings (14) provide initial support for this concept showing that treatment with drugs that improve cognitive function in aged mice also exerts positive effects on gait coordination. It is expected that research in this arena will exponentially expand in the upcoming years; thus methods to reliably characterize age- and pathology-related subclinical changes in mouse gait are very much needed.

It is an important strength of our study that we have developed an easy-to-implement method to characterize gait variability in mice. Healthy neural control systems can fine-tune the stride-tostride fluctuations of gait. Increased gait variability is a sensitive indicator, which in older humans was reported to predict cognitive decline $(22,23)$ and decreased survival (24), and associate with risk of falls (25). Our findings demonstrating increased gait variability in aged mice have therefore important translational relevance. In older adults, gait variability is thought to be associated with areas important for sensorimotor integration, coordination (17), and memory and executive function (26). There is strong evidence showing that a wide range of age-related pathologies increase gait variability. Recent magnetic resonance imaging studies conducted on elderly patients revealed an association of infarcts and white matter hyperintensities (a radiological sign of microvascular ischemia) with greater variability of spatial gait parameters (step length) (27).

Another important index of gait function that we introduce to geroscience research is the analysis of gait asymmetry. Although aging per se does not alter gait symmetry in mice, it is expected that this method will be particularly useful to assess longitudinal consequences of pathologies that show nonuniform distribution in the aged brain (eg, stroke, microhemorrhages, traumatic brain injury).

It is an important aspect of our study that we characterized agerelated changes in baseline gait performance in healthy mice voluntarily walking at a constant speed. In follow-up studies we will also determine age-related changes in mice during treadmill walking over a range of walking and running speeds and characterize alterations in gait coordination and cognition simultaneously in mouse models with accelerated aging (eg, high-fat-diet feeding) and age-related brain pathologies (Alzheimer's disease, hypertension-induced cerebral microhemorrhages).

In conclusion, male C57BL/6 mice exhibit quantifiable, clinically relevant alterations in gait function at 24 months of age. Our findings suggest that in addition to the commonly used temporal and spatial gait characteristics, gait variability and gait asymmetry are useful indices to characterize mouse models of aging and agerelated pathologies.

\section{Supplementary Material}

Supplementary data are available at The Journals of Gerontology, Series A: Biological Sciences and Medical Sciences online.

\section{Funding}

This work was supported by grants from the American Heart Association (AHA 17POST33661173 to ST) (S.T., Z.I.U., and A.C.), the Oklahoma Center for the Advancement of Science and Technology (to A.C., A.Y., and Z.I.U.), the National Center for Complementary and Alternative Medicine (R01-AT006526 to Z.I.U.), the National Institute on Aging (R01-AG055395, R01-AG047879, R01-AG038747, R01-AG055395), the National Institute of Neurological Disorders and Stroke (NINDS; R01-NS100782, R01-NS056218), the Oklahoma Shared Clinical and Translational Resources (OSCTR) program funded by the National Institute of General Medical Sciences (U54GM104938 to A.Y.), the Presbyterian Health Foundation (to Z.I.U., A.C., and A.Y.) and the EU-funded Hungarian grant (EFOP-3.6.1-162016-00008). The authors acknowledge the support from the NIA-funded Geroscience Training Program in Oklahoma (T32AG052363).

\section{Conflict of interest statement}

None declared.

\section{References}

1. Studenski S, Perera S, Patel K, et al. Gait speed and survival in older adults. JAMA. 2011;305:50-58. doi: 10.1001/jama.2010.1923

2. Sorond FA, Galica A, Serrador JM, et al. Cerebrovascular hemodynamics, gait, and falls in an elderly population: MOBILIZE Boston study. Neurology. 2010;74:1627-1633. doi: 10.1212/WNL.0b013e3181df0982

3. Sorond FA, Kiely DK, Galica A, et al. Neurovascular coupling is impaired in slow walkers: the MOBILIZE Boston study. Ann Neurol. 2011;70:213220. doi: 10.1002/ana.22433

4. Sorond FA, Cruz-Almeida Y, Clark DJ, et al. Aging, the central nervous system, and mobility in older adults: neural mechanisms of mobility impairment. $J$ Gerontol A Biol Sci Med Sci. 2015;70:1526-1532. doi: 10.1093/gerona/glv130

5. Tian Q, Bair WN, Resnick SM, Bilgel M, Wong DF, Studenski SA. $\beta$-Amyloid deposition is associated with gait variability in usual aging. Gait Posture. 2018;61:346-352. doi: 10.1016/j.gaitpost.2018.02.002 
6. Vestergaard S, Patel KV, Bandinelli S, Ferrucci L, Guralnik JM. Characteristics of 400-meter walk test performance and subsequent mortality in older adults. Rejuvenation Res. 2009;12:177-184. doi: 10.1089/rej.2009.0853

7. Mielke MM, Roberts RO, Savica R, et al. Assessing the temporal relationship between cognition and gait: slow gait predicts cognitive decline in the Mayo Clinic Study of Aging. J Gerontol A Biol Sci Med Sci. 2013;68:929_ 937. doi: 10.1093/gerona/gls256

8. Walston J, Hadley EC, Ferrucci L, et al. Research agenda for frailty in older adults: toward a better understanding of physiology and etiology: summary from the American Geriatrics Society/National Institute on Aging Research Conference on Frailty in Older Adults. J Am Geriatr Soc. 2006;54:991-1001. doi:10.1111/j.1532-5415.2006.00745.x

9. Pamoukdjian F, Paillaud E, Zelek L, et al. Measurement of gait speed in older adults to identify complications associated with frailty: a systematic review. J Geriatr Oncol. 2015;6:484-496. doi: 10.1016/j.jgo.2015.08.006

10. Richardson A, Fischer KE, Speakman JR, et al. Measures of healthspan as indices of aging in mice-a recommendation. J Gerontol A Biol Sci Med Sci. 2016;71:427-430. doi: 10.1093/gerona/glv080

11. Ungvari Z, Tarantini S, Hertelendy P, et al. Cerebromicrovascular dysfunction predicts cognitive decline and gait abnormalities in a mouse model of whole brain irradiation-induced accelerated brain senescence. Geroscience. 2017;39:33-42. doi: 10.1007/s11357-017-9964-z

12. Laufer Y. Effect of age on characteristics of forward and backward gait at preferred and accelerated walking speed. J Gerontol A Biol Sci Med Sci. 2005;60:627-632.

13. Błażkiewicz M, Wiszomirska I, Wit A. Comparison of four methods of calculating the symmetry of spatial-temporal parameters of gait. Acta Bioeng Biomech. 2014;16:29-35.

14. Tarantini S, Valcarcel-Ares NM, Yabluchanskiy A, et al. Treatment with the mitochondrial-targeted antioxidant peptide SS-31 rescues neurovascular coupling responses and cerebrovascular endothelial function and improves cognition in aged mice. Aging Cell. 2018;17. doi:10.1111/acel.12731.

15. Parks RJ, Fares E, Macdonald JK, et al. A procedure for creating a frailty index based on deficit accumulation in aging mice. J Gerontol A Biol Sci Med Sci. 2012;67:217-227. doi: 10.1093/gerona/glr193

16. Liu H, Graber TG, Ferguson-Stegall L, Thompson LV. Clinically relevant frailty index for mice. J Gerontol A Biol Sci Med Sci. 2014;69:1485-1491. doi: 10.1093/gerona/glt188

17. Tian Q, Chastan N, Bair WN, Resnick SM, Ferrucci L, Studenski SA. The brain map of gait variability in aging, cognitive impairment and dementia- a systematic review. Neurosci Biobehav Rev. 2017;74(Pt A):149-162. doi: 10.1016/j.neubiorev.2017.01.020

18. Sudarsky L. Gait disorders: prevalence, morbidity, and etiology. Adv Neurol. 2001;87:111-117.

19. Toth P, Tarantini S, Csiszar A, Ungvari Z. Functional vascular contributions to cognitive impairment and dementia: mechanisms and consequences of cerebral autoregulatory dysfunction, endothelial impairment, and neurovascular uncoupling in aging. Am J Physiol Heart Circ Physiol. 2017;312:H1-H20. doi: 10.1152/ajpheart.00581.2016

20. Tarantini S, Yabluchanksiy A, Fülöp GA, et al. Pharmacologically induced impairment of neurovascular coupling responses alters gait coordination in mice. Geroscience. 2017;39:601-614. doi: 10.1007/ s11357-017-0003-x

21. Tarantini S, Valcarcel-Ares NM, Yabluchanskiy A, et al. Insulin-like growth factor 1 deficiency exacerbates hypertension-induced cerebral microhemorrhages in mice, mimicking the aging phenotype. Aging Cell. 2017;16:469-479. doi: 10.1111/acel.12583

22. Decker LM, Cignetti F, Hunt N, Potter JF, Stergiou N, Studenski SA. Effects of aging on the relationship between cognitive demand and step variability during dual-task walking. Age (Dordr). 2016;38:363-375. doi: 10.1007/s11357-016-9941-y

23. Herman T, Giladi N, Gurevich T, Hausdorff JM. Gait instability and fractal dynamics of older adults with a "cautious" gait: why do certain older adults walk fearfully? Gait Posture. 2005;21:178-185. doi: 10.1016/j.gaitpost.2004.01.014

24. Verlinden VJ, van der Geest JN, Hoogendam YY, Hofman A, Breteler MM, Ikram MA. Gait patterns in a community-dwelling population aged 50 years and older. Gait Posture. 2013;37:500-505. doi: 10.1016/j. gaitpost.2012.09.005

25. Verghese J, Ambrose AF, Lipton RB, Wang C. Neurological gait abnormalities and risk of falls in older adults. J Neurol. 2010;257:392-398. doi: 10.1007/s00415-009-5332-y

26. Rosso AL, Olson Hunt MJ, Yang M, et al.; Health ABC study. Higher step length variability indicates lower gray matter integrity of selected regions in older adults. Gait Posture. 2014;40:225-230. doi: 10.1016/j. gaitpost.2014.03.192

27. Rosano C, Brach J, Studenski S, Longstreth WT Jr, Newman AB. Gait variability is associated with subclinical brain vascular abnormalities in high-functioning older adults. Neuroepidemiology. 2007;29:193-200. doi: $10.1159 / 000111582$ 